\title{
Familiarity and recognition of faces in old age
}

\author{
JAMES C. BARTLETT and ANNETTE FULTON \\ University of Texas at Dallas, Richardson, Texas
}

\begin{abstract}
Elderly persons exceed young adults in false recognitions of new faces. One account claims there are age-related deficits in memory for context of encounter with faces. Because of these deficits, elderly persons frequently recognize faces on the basis of perceived familiarity (i.e., resemblance to face representations in memory), which is high for some new faces. To test this contextrecollection hypothesis, we had young adult and elderly subjects judge whether faces: (1) had been seen previously in a test (though no face was repeated), and (2) were subjectively familiar (though no face was famous). The elderly exceeded the young subjects in seen-before judgments (false recognitions), and only the elderly showed a positive correlation between false recognitions and subjective familiarity. In Experiment 2, this finding was extended from false recognitions to correct recognitions, supporting the view that elderly persons, compared to young adults, rely more on resemblance in recognizing faces.
\end{abstract}

Recognition memory is quite good in old age (Craik, 1977; Craik \& McDowd, 1987). However, there are agerelated differences in the recognition of faces (Bartlett \& Leslie, 1986; Bartlett, Leslie, Tubbs, \& Fulton, 1989; Ferris, Crook, Clark, McCarthy, \& Rae, 1980; Smith \& Winograd, 1978). These differences show a characteristic pattern of near age-invariance in hits in response to old faces and an age-related increase in false alarms in response to new faces (Bartlett et al., 1989; Bartlett, Strater, \& Fulton, 1991). No existing theory predicts such a pattern, but contemporary models of face recognition (Bruce, 1988) suggest some testable accounts. The goal in this study, as well as in a previous investigation (Bartlett et al., 1991), was to advance our understanding of both cognitive aging and face processing by testing among these theoretical accounts.

In our previous paper (Bartlett et al., 1991), we used the face-processing model of Bruce and Young (1986) as a theoretical framework. According to this model, face recognition involves a decision-making system that weighs information from (at least) two retrieval processes. One process signals the degree of resemblance between a face that is currently being examined and representations of faces in memory. The other process accesses semantic information specifying the identity of a face and/or contextual information about the circumstances of encounter with this face (see, e.g., Brown, Deffenbacher, \& Sturgill, 1977; Hanley \& Cowell, 1988; Young, Hay, \& Ellis, 1985). The distinction between semantic and contextual

This project was supported by National Institute on Aging Grant RO1AG07798. We thank Hervé Abdi, Alice O'Toole, and W. Jay Dowling for helpful discussions concerning the research, and Peggy IntonsPeterson, Fergus Craik, and Andy Young for extensive and thoughtful comments on an earlier version of the article. Correspondence should be sent to James Bartlett, Program in Human Development and Communication Sciences, University of Texas at Dallas, Box 830688 , Richardson, TX 75080-0688. information is subtle with faces, because much of what we know about a great many faces involves when and where these faces are seen (Bruce, 1988). Bartlett et al. (1991) focused on contextual information, but we made no claims about its plausible relation to semantic information.

Within the framework of the Bruce and Young (1986) model, age-related differences in false recognitions of new faces might be due to underlying differences in the decision-making process (e.g., older subjects might adopt more lax decision criteria), in the resemblance process (perceived resemblance of new faces might be increased in old age), and/or in the context-retrieval process (older subjects might be less able or less likely to perform such retrieval). To evaluate these three possibilities, Bartlett et al. (1991) compared young adult and elderly subjects in two face-recognition tasks: (1) that of judging whether or not faces were recent (i.e., seen within the last hour), and (2) that of judging whether or not faces were famous (i.e., prevalent in the media). We found that false recent judgments and false famous judgments showed age-related increases, and that these age-related increases were larger for lures that had been viewed 1 week previously than they were for entirely new lures that had not been seen before. The pattern suggested that the elderly were deficient at context recollection, or simply failed to engage in context recollection, so that their recognition judgments were significantly affected by the level of resemblance of items on the test.

Although the findings obtained by Bartlett et al. (1991) supported age differences in context recollection, it remained to be shown that age-related increases in false recognitions of new faces-faces that subjects have never seen before-involve context recollection. False alarms in response to new faces are quite poorly understood (Davies, Shepherd, \& Ellis, 1979; Goldstein, Stephenson, \& Chance, 1977), and we know of no evidence that context recollection affects such false alarms. Hence, whether age-related differences in context recollection could ac- 
count for age differences in responses to new faces was an entirely open question.

Our approach to this question was based on a study (Vokey \& Read, 1988) in which subjects saw a sequence of nonfamous faces that they had never seen before. Although no face was repeated within the sequence, the subjects were not informed of this fact. They were simply told to respond to each face with: (1) a recognition judgment (Was the face presented earlier in the sequence?), (2) a subjective familiarity judgment (Does the face appear confusable with someone known from life?), and (3) a typicality judgment (Is the face typical in the sense that it would be hard to spot in a crowded train station; see Valentine \& Bruce, 1986). ${ }^{1}$

For purposes of the present research, the most relevant findings of Vokey and Read (1988) concerned correlations between each pair of judgments across the 209 face stimuli. Typicality was correlated with false recognitions as in several prior studies (Going \& Read, 1974; Light, Kayra-Stuart, \& Hollander, 1979). Moreover, typicality was correlated with subjective familiarity as in Bartlett, Hurry, and Thorley (1984). The major new finding was that false recognitions and subjective familiarity judgments were not correlated with each other $(r s=.11$ and .02 for male and female subjects, respectively).

If one assumes that separate processes occur for perception of resemblance and retrieval of context, the lack of a recognition/familiarity correlation can easily be explained. Presumably, Vokey and Read's (1988) subjects frequently detected resemblances between faces encountered in the test and (1) faces seen earlier in the test, as well as (2) faces encountered in everyday life. However, they recollected contextual information in order to attribute these resemblances to their appropriate source (the experiment or life). Hence, faces that resembled other faces in the test received more false recognitions, but not more judgments of subjective familiarity, than did faces that did not. Likewise, faces that resembled faces seen in life received more judgments of subjective familiarity, but not more false recognitions, than did faces that did not. Although both false recognitions and subjective familiarity were positively correlated with face typicality, these correlations were apparently too weak (all $r s<.40$ ) to produce, by themselves, significant recognition/familiarity correlations.

Vokey and Read (1988) appear to have used only young adults in their study. This raises the question of what might be found if elderly subjects were employed. If the elderly are deficient in recollecting context, or if they simply do not engage in context retrieval when making recognition and familiar judgments, they should be subject to two types of confusion: First, the perception of resemblance between a face on the test and a face from real life might be attributed mistakenly to a match with a prior face on the test. Second, the perception of resemblance between a face on the test and a prior test face might be attributed mistakenly to a match with a face from real life. These misattributions would produce a correlation between subjective familiarity judgments and false recognitions. Thus, we can predict that elderly subjects, unlike young adults, should show a positive correlation between subjective familiarity and false recognitions.

The prediction of the context-recollection hypothesis conflicts with that of a plausible alternative. A number of studies of word and sentence memory have supported the existence of age-related deficits in distinctiveness of encoding (Craik \& Simon, 1980). For example, the elderly tend to exceed young adults in false alarms in response to lures chosen to be similar to previously studied words (e.g., synonyms of input items; see Rankin \& Kausler, 1979; Smith, 1975). Such findings suggest an age-related deficit in the forming of memory traces that are sufficently specific to distinguish exact copies of original stimuli from subtly different lures. Such an agerelated deficit in distinctiveness of encoding might well generalize to faces. When older persons attend to a face, they might encode relatively few facial features, or they might encode relatively nondistinguishing features-that is, features that are shared by a number of faces and that do not uniquely specify any one face (Winograd, 1981). In either case, the memory traces that are formed by older subjects would tend to "match" new items presented subsequently in a test, causing these items to have high perceived resemblance. This would cause older subjects to make more false alarms, compared to young adults.

Unlike the context-recollection hypothesis, the encodingdistinctiveness hypothesis makes no predictions regarding correlations between subjective-familiarity judgments and false recognitions. In light of the findings of Vokey and Read (1988), one could only expect that such correlations should be small and nonsignificant regardless of age group. However, given prior evidence that typical faces evoke more false alarms than unusual faces do (see, e.g., Light et al., 1979), it is a reasonable assumption that nondistinctive encoding should lead to high rates of falsealarm errors primarily in response to typical new items, as opposed to unusual new items. Given this assumption, the encoding-distinctiveness hypothesis predicts age differences in false-alarm errors to be larger in response to typical faces than in response to distinctive faces. Put differently, elderly subjects should show stronger correlations between typicality ratings and false recognitions than young adults do.

\section{EXPERIMENT 1}

In a task like that of Vokey and Read (1988), young adult and elderly subjects viewed a sequence of faces, judging each item for subjective familiarity (Is it confusable with someone you know?) and episodic recognition (Has this face been seen earlier in the sequence?). The subjects then viewed the same sequence again, this time making ratings of face typicality. We expected that elderly subjects would make greater numbers of (false) 
recognitions than young adults would. Our main questions were whether: (1) the correlation between subjectivefamiliarity judgments and false recognitions would be significantly positive in the elderly group but not the young group, as the context-recollection hypothesis predicts, or (2) the correlation between typicality and false recognitions would be stronger in the elderly group than in the young group, as the encoding-distinctiveness hypothesis predicts.

A potential criticism of the present investigation, as well as the study by Vokey and Read (1988), is that subjectivefamiliarity judgments in response to strangers' faces are entirely inconsistent and idiosyncratic. Indeed, it is doubtlessly true that certain faces seem familiar to only a subset of observers (who have a friend or relative with a similar appearance), yet strike other persons as entirely unfamiliar. This point notwithstanding, it is a plausible assumption that at least some faces are consistently judged as more familiar than others, at least for subjects in a certain age group (e.g., a face resembling that of a famous politician might seem familiar across subjects). The testing of this assumption is critical to our empirical approach of assessing correlations of subjective familiarity with other attributes of faces. Hence, we asked our young and elderly subjects to judge young, middle-aged, and elderly faces. Assuming that people have more friends and acquaintances of about their same ages than they do ones who are younger or older, we made the following prediction: Young faces would receive more familiar judgments from young subjects than from elderly subjects, whereas elderly faces would receive more familiar judgments from elderly subjects than from young subjects. If this prediction should be confirmed, it would support the validity of subjective familiarity judgments.

\section{Method}

Subjects. The 9 young men and 10 young women participants ranged in age from 22 to 43 years ( $M=30$ years); the 10 elderly men and 9 elderly women ranged in age from 59 to 71 years $(M=64$ years). The young subjects were undergraduate psychology students at the University of Texas at Dallas, who participated as one alternative means of fulfilling a course requirement. The elderly were recruited from church and community groups in the Dallas area, and they participated for pay $(\$ 10)$. All of the elderly were high school graduates, and 12 had completed 1 or more years of college ( 5 were college graduates). Scores on a vocabulary test (the 20 words of the second half of the WAIS were used) averaged 25.3 versus 25.4 out of 40 ( 20 words at 2 points per word) for young and elderly subjects, respectively ( $S D s=7.1$ vs. 8.9 , respectively). Corrected visual acuity, tested with a Snellen chart, was $20 / 40$ or better in the best eye. All subjects reported that they were in good health.

Materials. All stimuli were $35-\mathrm{mm}$ color slides, each showing a head-and-shoulders, right or left three-quarter view of a face either smiling or with neutral expression. No jewelry was visible, and clothing was obscured by a white sheet draped around the shoulders.

Since we wanted to examine effects of face age, and since the true ages of some faces were unknown, we had 10 young adult subjects rate the age of each face. On the basis of these ratings, we divided the 72 test faces into young, middle-aged, and elderly subsets (mean rated ages $=31,50$, and 65 years, respectively), with each set including 12 male and 12 female faces.

The test sequence was a random ordering of the 72 test faces, with 12 filler faces added at the start. The ordering of the 72 criti- cal test faces, but not of the 12 fillers, was reversed for half of the young and elderly subjects. The 12 fillers included 6 male faces and 6 female faces, and 5 young, 2 middle-aged, and 5 elderly faces (mean rated ages $=23,42$, and 61 years, respectively).

Procedure. The 3-5 subjects in each experimental session first completed a short questionnaire on health and educational background. Second, their visual acuity was individually tested. Third, they took a 20 -item vocabulary test for which $10 \mathrm{~min}$ were allowed. Fourth, the subjects were told that they would see 84 faces, and that their task would be to respond to each face by judging: (1) whether it had occurred earlier in the sequence, possibly in a different pose or with a different expression, and (2) whether it was confusable with a face of someone they knew or had seen in everyday life. In fact, no face was repeated within the test, and, to the best of our knowledge, none were previously known to the subjects. The statement that faces might be repeated with changes was intended to ensure that there would be substantial numbers of false recognitions.

The 84-item test was presented via slide projector with 10-sec presentations of each face and a 3-sec response interval following each presentation. The subjects responded to each item by circling " $Y$ " (for yes) or " $N$ " (for no) in each of two columns, one labeled "familiar?," and another labeled "recognize?"

In the next phase, the subjects were told that they would see the sequence of faces again, but that this time their task was to rate each face on a 7-point scale from highly typical (1) to highly distinctive or unusual (7). To clarify the task, the subjects were asked to "imagine you are supposed to meet each of these people at the airport. Rate how distinctive or unusual each face is based on how easy you think it would be to pick the person out in a crowd. If the face is very distinctive and would be easy to spot in a crowd, circle 7. If the face is very typical and thus hard to spot in a crowd, circle 1 . For faces that fall between most unusual and most typical, circle 6, 5, 4, 3, or 2" (cf. Valentine \& Bruce, 1986).

After having received these instructions, the subjects viewed the 84-item sequence under the same conditions as before. They indicated each of their face-typicality judgments by circling a number from 1 through 7 on their response sheets. ${ }^{2}$

\section{Results and Discussion}

Effects of age of subject and test block. We first examined age differences in false recognitions (Figure 1) and subjective-familiarity judgments (Figure 2 ) in successive 12-item blocks of the test (the first block contained the 12 filler items). An analysis of variance (ANOVA)

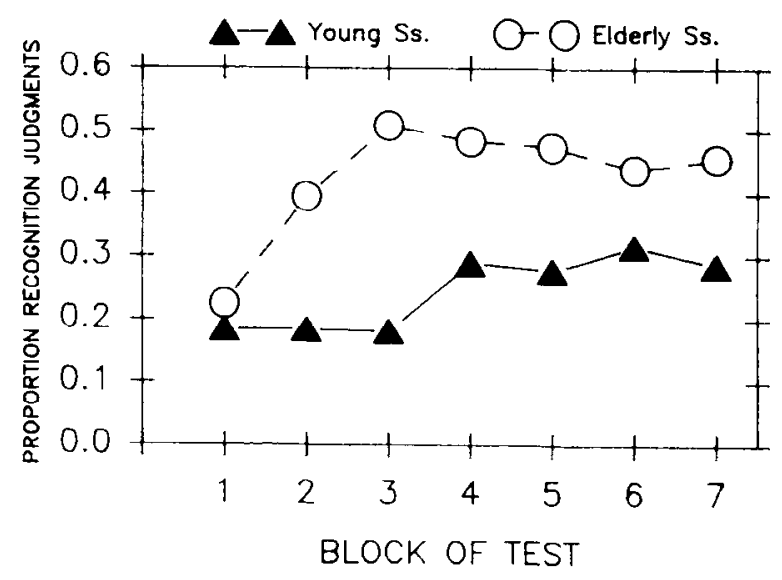

Figure 1. Mean proportions of false recognition judgments made by young and elderly subjects in each 12-item test block in Experiment 1. The first block contained the 12 fller items. 


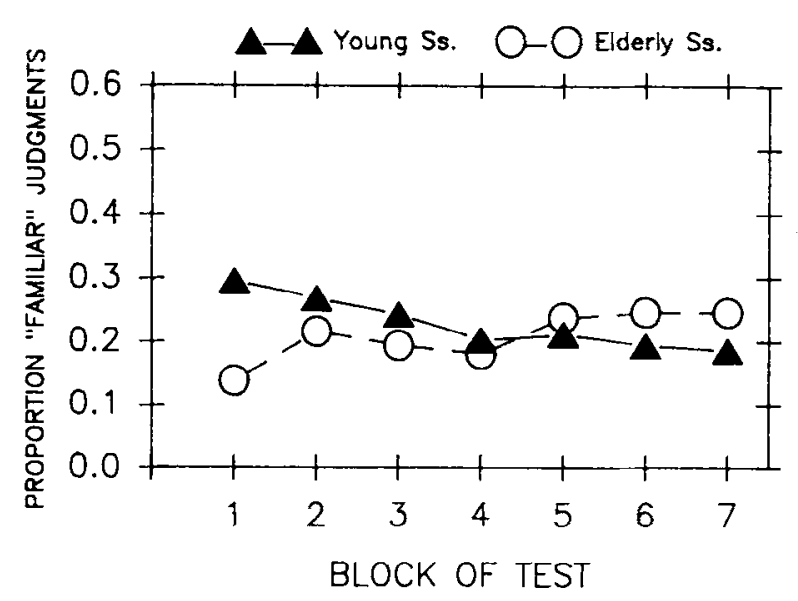

Figure 2. Mean proportions of familiar judgments made by young and elderly subjects in each 12-item test block in Experiment 1. The first block contained the 12 filler items.

of the false-recognition data showed a main effect for age of subject $\left[F(1,34)=12.6, M S_{e}=.168\right]$, a main effect for test block $\left[F(6,204)=10.3, M S_{e}=.017\right]$, and an age-of-subject $\times$ test-block interaction $[F(6,204)=4.3$, $\left.M S_{e}=.017\right]$, with no effects involving sex of subject. There was essentially no age difference in the first test block $[t(36)<1]$, but false recognitions by the elderly subjects reliably exceeded those by the young in all other test blocks except Test Block $6[t \mathrm{~s}(36)=3.97,5.61,3.16$, $3.03,1.66$, and 2.31 for Blocks 2-7, respectively]. For our chosen alpha level of .05, the critical $t$ value was 2.02 .

An ANOVA of proportions of subjects' familiar judgments supported only an age-of-subject $\times$ test-block interaction $\left[F(6,204)=4.28, M S_{\mathrm{e}}=.013\right]$. Familiar judgments showed a significant age difference only in Block 1 of the test $[t(36)=2.25]$, but they fell with test position in the young adult group $\left[F(6,108)=2.53, M S_{e}=.013\right]$ and rose with test position in the elderly group $[F(6,108)$ $=2.60, M S_{\mathrm{e}}=.012 \mathrm{]}$. We will return to this finding in the General Discussion.

Effects of age of subject and age and sex of face. In a second set of ANOVAs, we omitted the variable of test block in order to examine the effects of age of face and sex of face. The 12 filler items (Block 1 in Figures 1 and 2) were dropped from these ANOVAs, which were performed first with subjects treated as the random factor and then with faces treated as the random factor. In Table 1 , the effects that were significant in both the subjectsrandom and the faces-random tests are listed.

The ANOVAs performed on false-recognition judgments supported only the age-of-subject effect. In contrast, the ANOVAs of subjective-familiarity judgments showed significant interactions between age of subject and age of face, and between sex of face and age of face (see Figure 3). In general accordance with our predictions, young subjects exceeded elderly subjects in familiar judgments in response to young faces, whereas elderly subjects exceeded young subjects in familiar judgments in response to middle-aged and elderly faces (Figure 3, left panel). A minor puzzle is that although the proportions of familiar judgments among young subjects decreased as a function of face age (as one would predict), the proportions of familiar judgments among elderly subjects were as high for middle-aged faces as for elderly faces, if not higher. In addition, an unexpected effect was that familiar judgments in response to young faces were more frequent if these faces were female, whereas familiar judgments in response to elderly faces were more frequent if these faces were male (Figure 3, right panel). ${ }^{3}$

Another unexpected result was found in the typicality ratings. There was a main effect of age of face, qualified by interactions with sex of subject and with both sex and age of subject (Table 1). Younger faces were rated as more typical (i.e., less distinctive), and this effect appeared strongest among elderly male subjects, and weakest among elderly female subjects (Table 2 ). ${ }^{4}$

In summary, false recognitions but not familiar judgments showed an age-related increase (Figures 1 and 2), whereas familiar judgments but not false recognitions were more frequent in response to faces in the same age range as subjects, and also to young female and old male faces (Figure 3). Typicality judgments differed from both other types of judgment in showing a face-age effect that varied by sex of subject as well as age of subject. Although

Table 1

Outcomes of ANOVAs Performed on False-Recognition Judgments, Subjective-Familiarity Judgments, and Distinctiveness Ratings from Experiment 1

\begin{tabular}{|c|c|c|c|c|c|c|}
\hline \multirow{2}{*}{$\begin{array}{c}\text { Measure and } \\
\text { Independent Variable }\end{array}$} & \multicolumn{3}{|c|}{ Subjects Random } & \multicolumn{3}{|c|}{ Items Random } \\
\hline & $d f$ & $F$ & $\overline{M S_{\mathrm{e}}}$ & $d f$ & $F$ & $\overline{M S_{\mathrm{e}}}$ \\
\hline $\begin{array}{l}\text { False recognition } \\
\text { Age of subject }\end{array}$ & 1,34 & 13.8 & .162 & 1,66 & 110.1 & .026 \\
\hline $\begin{array}{l}\text { Subjective familiarity } \\
\text { Age of face } \times \text { age of subject } \\
\text { Age of face } \times \text { sex of face }\end{array}$ & $\begin{array}{l}2,68 \\
2,68\end{array}$ & $\begin{array}{l}3.2 \\
5.0\end{array}$ & $\begin{array}{l}.016 \\
.013\end{array}$ & $\begin{array}{l}2,66 \\
2,66\end{array}$ & $\begin{array}{l}5.3 \\
3.3\end{array}$ & $\begin{array}{l}.016 \\
.026\end{array}$ \\
\hline $\begin{array}{l}\text { Typicality } \\
\text { Age of face } \\
\text { Age of face } \times \text { sex of subject } \\
\text { Age of face } \times \text { sex of subject } \times \text { age of subject }\end{array}$ & $\begin{array}{l}2,68 \\
2,68 \\
2,68 \\
\end{array}$ & $\begin{array}{r}26.3 \\
4.2 \\
6.2 \\
\end{array}$ & $\begin{array}{l}.364 \\
.364 \\
.364 \\
\end{array}$ & $\begin{array}{l}2,66 \\
2,66 \\
2,66 \\
\end{array}$ & $\begin{array}{r}5.3 \\
7.4 \\
12.2 \\
\end{array}$ & $\begin{array}{r}2.301 \\
.276 \\
.233 \\
\end{array}$ \\
\hline
\end{tabular}

Note-Listed are the effects that gave $p<.05$ both with subjects random and with items random. 


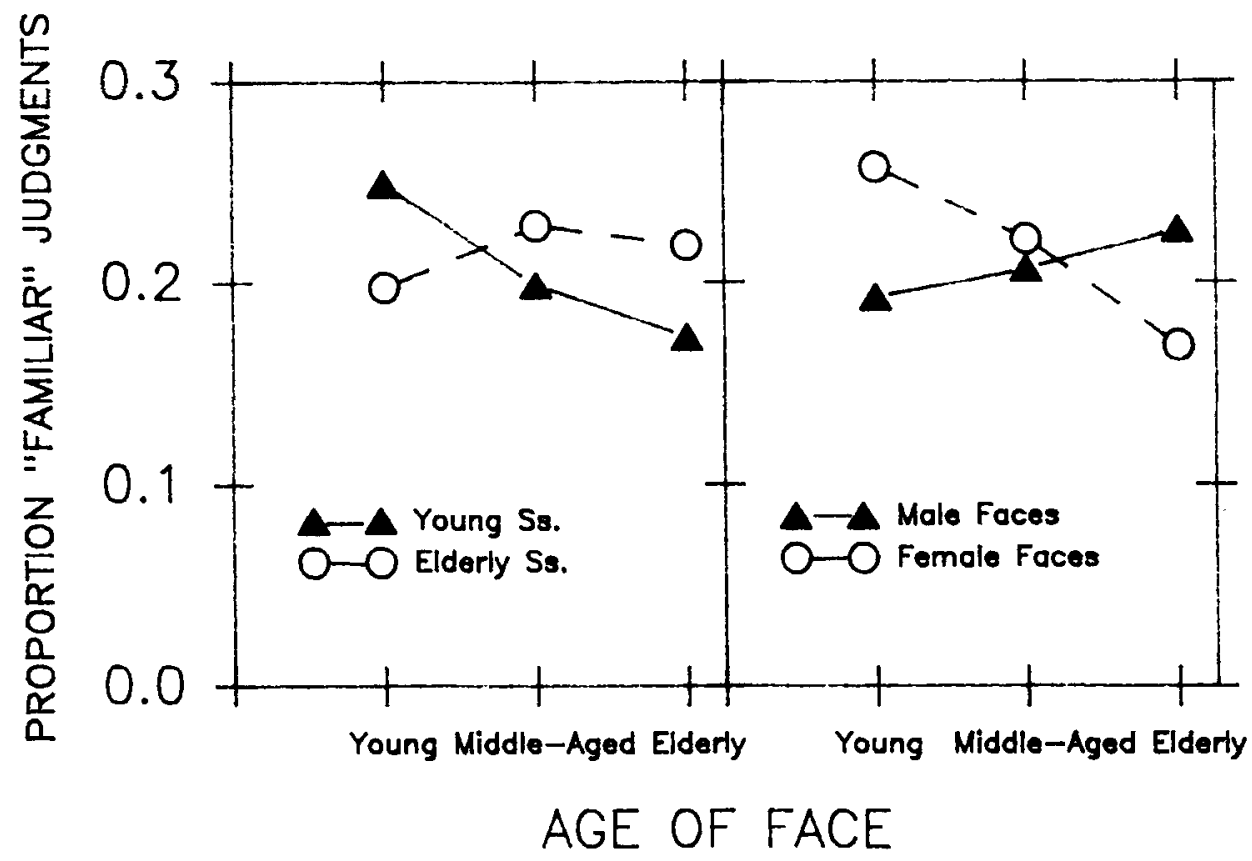

Figure 3. Mean proportion of familiar judgments by face age and subject age (left panel), and by face age and face sex (right panel) in Experiment 1.

we did not predict all of these findings, the data clearly show that the three types of judgments were based on different information. Moreover, the pattern of subjects' familiar judgments (Figure 3) supports our assumption that subjective familiarity was to some degree consistent across the subjects in each age group.

Correlational analyses. The responses made by young male subjects were used to derive three scores for each test item; a false-recognition rate, a subjectivefamiliarity-judgment rate, and a mean typicality rating. Similarly, the responses made by young female subjects, elderly male subjects, and elderly female subjects were used to derive three scores for each item. As in the Vokey and Read (1988) study, the typicality scores for male versus female subjects were strongly correlated $(r s=.80$ and .63 in the young and elderly groups, respectively). The false-recognition scores were moderately correlated ( $r \mathrm{~s}=.46$ and .42 , respectively), and the familiarjudgment scores were very weakly correlated $(r s=.31$ and .16, respectively). The very small size of the latter correlations raises some interpretive issues that we will consider shortly. ${ }^{5}$

The main predictions for this study concern correlations between typicality and subjective-familiarity scores, typicality and false-recognition scores, and subjectivefamiliarity and false-recognition scores. In Table 3, these three correlations are displayed for each combination of subject age and subject sex, along with the data from Vokey and Read (1988). In Table 4 (two left columns) are displayed the correlations that emerged when we collapsed the data from male and female subjects in deriving the measures for each test item.
A discrepancy from the Vokey and Read (1988) study was that there were no significant correlations between typicality and subjective familiarity. However, our young subjects replicated Vokey and Read's findings of: (1) significant correlations between typicality and false recognitions, and (2) minimal correlations between subjective familiarity and false recognitions. Our elderly subjects were similar in showing significant typicality/recognition correlations. However, the elderly differed from the young adult subjects in showing significantly positive correlations between subjective familiarity and false recognitions-the corresponding correlations among young subjects were not only nonsignificant, but negative in sign. Thus, faces more frequently judged as familiar were also more frequently judged to be old (i.e.,

Table 2

Mean Typicality Ratings in Response to Presentations of Young Middle-Aged, and Elderly Faces by Age and Sex of Subject in Experiment 1

\begin{tabular}{|c|c|c|c|c|c|c|c|}
\hline \multirow{3}{*}{$\begin{array}{l}\text { Age and Sex } \\
\text { of Subjects }\end{array}$} & \multirow[b]{3}{*}{$n$} & \multicolumn{6}{|c|}{ Age of Face } \\
\hline & & \multicolumn{2}{|c|}{ Young } & \multicolumn{2}{|c|}{ Middle } & \multicolumn{2}{|c|}{ Elderly } \\
\hline & & $M$ & $S D$ & $M$ & $S D$ & $M$ & $S D$ \\
\hline \multicolumn{8}{|l|}{ Young } \\
\hline Males & 9 & 4.67 & .73 & 4.64 & .76 & 4.17 & .88 \\
\hline Females & 10 & 4.88 & .99 & 4.73 & .98 & 4.25 & .83 \\
\hline \multicolumn{8}{|l|}{ Elderly } \\
\hline Males & 10 & 5.34 & .44 & 4.53 & .51 & 3.86 & .48 \\
\hline Females & 9 & 4.29 & .69 & 4.03 & .70 & 4.06 & .95 \\
\hline
\end{tabular}

Note-The 7-point typicality scale has been inverted, so that $7=\operatorname{mos}$ typical and $1=$ most distinctive. 
Table 3

Correlations among Typicality, Subjective-Familiarity, and False-Recognition Judgments for Male and Female Subjects in Vokey and Read (1988) and in Both Age Groups of Experiment 1

\begin{tabular}{|c|c|c|c|c|c|c|}
\hline \multirow[b]{3}{*}{ Correlation } & & & \multicolumn{4}{|c|}{ Experiment 1} \\
\hline & \multicolumn{2}{|c|}{ Vokey and Read } & \multicolumn{2}{|c|}{ Young Subjects } & \multicolumn{2}{|c|}{ Elderly Subjects } \\
\hline & Male & Female & Male & Female & Male & Female \\
\hline Typicality/familiarity & $.26^{*}$ & $.39 *$ & -.02 & .07 & .13 & .06 \\
\hline Typicality/recognition & $.33^{*}$ & $.31^{*}$ & $.27^{*}$ & $.38^{*}$ & $.38^{*}$ & $.24^{*}$ \\
\hline Familiarity/recognition & .11 & .02 & -.06 & -.15 & $.26^{*}$ & $.30 *$ \\
\hline
\end{tabular}

Note-Degrees of freedom $=207$ in Vokey and Read (1988) and 70 in Experiment 1. The subjects in the Vokey and Read study were college undergraduates and presumably were young. ${ }^{*} p<.05$.

seen before in the test), but only among the elderly subjects. This was precisely the pattern predicted by the context-recollection hypothesis.

Although the age differences in familiarity/recognition correlations were consistent with predictions, the very weak correlations between males' familiar judgments and females' familiar judgments ( $r=.16$ in the elderly group) resurrected a concern with which we started this research: Perhaps subjects' familiar judgments were simply too inconsistent and idiosyncratic to have meaningful correlations with anything else. Some degree of consistency in subjects' familiar judgments was supported by our findings that subject age, face age, and face sex entered into significant effects on these judgments (Figure 3 ). To gain more evidence on this point, we attempted to identify subsets of our faces that were consistently judged as familiar or not by male versus female subjects, and to examine the familiarity/recognition correlations within these sets of consistently judged items.

Our procedure was the following: For each of the 72 faces, we computed the absolute value of the difference between the proportion of male subjects in a given age group who made a familiar judgment and the proportion of female subjects in that age group who made a familiar judgment. We then selected those faces with difference scores at or below the median, arriving at a set of 34 faces that were consistently judged by young subjects, and an overlapping set of 38 faces that were consistently judged by elderly subjects. Across the faces in these two sets, subjective-familiarity judgments made by males versus females were very highly correlated $(r=.81$ for the young adult set, and .87 for the elderly set). And Table 4 shows that, within these sets of consistently judged faces, the

Table 4

Correlations among Typicality, Subjective-Familiarity, and False-Recognition Judgments Made by Young and Elderly Subjects for All Faces and for Faces Judged Consistently in Subjective Familiarity in Experiment 1

\begin{tabular}{lccccc}
\hline & \multicolumn{2}{c}{ All Faces } & & \multicolumn{2}{c}{$\begin{array}{c}\text { Consistently } \\
\text { Judged Faces }\end{array}$} \\
\cline { 2 - 3 } \cline { 5 - 6 } Correlation & $\begin{array}{c}\text { Young } \\
(n=72)\end{array}$ & $\begin{array}{c}\text { Elderly } \\
(n=72)\end{array}$ & $\begin{array}{c}\text { Young } \\
(n=34)\end{array}$ & $\begin{array}{c}\text { Elderly } \\
(n=38)\end{array}$ \\
\hline Typicality/familiarity & .03 & .10 & -.02 & .23 \\
Typicality/recognition & $.42 \dagger$ & $.33 \dagger$ & & $.41^{*}$ & $.37^{*}$ \\
Familiarity/recognition & -.16 & $.37 \dagger$ & -.19 & $.42 \dagger$ \\
\hline
\end{tabular}

${ }^{*} p<.05 . \quad \dagger p<.01$. correlations among subjective familiarity, false recognitions, and typicality were similar to those based on all 72 items despite the reduced $n \mathrm{~s}$. We conclude that there were subsets of our faces that evoked consistent judgments of subjective familiarity. And, within these subsets, our prediction for this study-an age difference in the familiarity/recognition correlation-was supported once again.

\section{EXPERIMENT 2}

Although the findings at this point appeared to be clear cut, it seemed essential to learn if the subjective-familiarity judgments of Experiment 1 would show a correlation with false recognitions by a different sample of elderly subjects in a new experiment. Assuming that a crossexperiment correlation would be found, we wanted to test an additional prediction of the context-recollection hypothesis. If elderly subjects rely heavily on resemblance in making recognition judgments, then subjectivefamiliarity should be positively correlated not only with false recognitions of faces (when they are entirely new to subjects), but also with correct recognitions of faces (when they have been previously viewed by subjects). That is, faces more frequently judged as familiar by elderly subjects in Experiment 1 should be recognized more often by another group of elderly subjects. Moreover, this finding should hold regardless of whether the faces are targets (previously viewed items) or lures (new items).

Although the basic prediction is straightforward, the findings might depend on the recognition task. In most prior studies on memory for faces, pictures of faces have been used as input items, and exact copies of these pictures have been used as recognition targets. Such tasks of picture recognition obviously might differ in their processing requirements from tasks of face recognition, in which persons are required to recognize faces despite visual changes from their input presentation (Bartlett \& Leslie, 1986; Hay \& Young, 1982).

To compare picture and face recognition, we used a procedure like that of Bartlett et al. (1989). An input list of pictures of faces was followed by a test that included identical copies of input face pictures, changed-expression versions of input face pictures, and entirely new face pictures. The recognition task was to judge each item as same, old-but-changed, or new. Proportions of same judg- 
ments in response to each item type can be viewed as proportions of picture-recognition judgments. If the proportions of new judgments in response to each item type are subtracted from unity (1.0), they can be viewed as proportions of face-recognition judgments. Of principal interest was whether the subjective-familiarity judgments from Experiment 1 would be correlated with proportions of picture-recognition judgments (hit and false-alarm rates), and/or with proportions of face-recognition judgments (hit and false-alarm rates), among elderly subjects.

\section{Method}

Subjects. Subjects were recruited as in Experiment 1. The 14 young men and 20 young women ranged in age from 22 to 42 years $(M=32$ years), and the 16 elderly men and 20 elderly women ranged in age from 59 to 79 years ( $M=69$ years). All of the young subjects were currently in college, 33 of the 36 elderly subjects had attended some college, and 14 of the elderly were college graduates. Vocabulary-test scores (second half of the WAIS) averaged 28.6 versus 27.6 out of 40 in the young and elderly groups, respectively $(S D s=6.2$ and 7.8 , respectively). Visual acuity (tested by Snellen chart) was 20/40 or better in the best eye (only 2 of the elderly scored worse than 20/30). All subjects reported that they were in good health.

Materials. The most important changes from Experiment 1 pertained to presentation of an input list prior to the recognition test. The input list contained 48 face pictures, plus 2 filler pictures at the beginning and end. The subsequent test contained 72 face pictures. These were the same 72 face pictures that made up the test of Experiment 1.

Of the 72 items on the recognition test, 24 were identical to inputlist items, 24 were old faces with facial expression changed (smile to neutral or vice versa), and 24 were entirely new faces. Three different versions of the input list were used with different subsets of young and elderly subjects. These three versions varied in the faces that they contained and in the expression of these faces, so that each face was tested equally often as an identical item, a changed-expression item, and an entirely new item.

Procedure. The sessions including 3-4 subjects began with the general information questionnaire, visual acuity testing, and vocabulary testing, as in Experiment 1. The subjects were then told that they would see a sequence of faces, and that their task was to look at each face for its entire 10-sec duration and then rate it for pleasantness on a 5-point scale $(1=$ most pleasant $)$ in the 3 -sec interval that followed. No mention was made of a memory test.

Immediately after the input list, the subjects were told that they would see another sequence of faces, and that their task was to judge each item as same (identical to an input face picture), old-butdifferent" (changed in expression from an input face picture), or new. They were shown example pictures of the same face with smiling and neutral expressions, and then the test sequence was started. Each face picture was shown for $10 \mathrm{sec}$ with a 3-sec response interval following.

\section{Results}

Table 5 displays the mean proportions of picturerecognition judgments, as well as mean proportions of face-recognition judgments (i.e., 1 minus mean proportions of new judgments) made by young and elderly subjects in response to identical, changed-expression, and new items at test. Both the picture- and face-recognition proportions were subjected to ANOVAs, including the
Table 5

Mean Proportions of Picture- and Face-Recognition Judgments Made by Young and Elderly Subjects in Response to Presentations of Identical, Changed-Expression, and New Faces in the Recognition Test of Experiment 2

Judgment Type and Subject Age

\begin{tabular}{|c|c|c|c|c|c|c|c|c|}
\hline \multirow[b]{4}{*}{ Item Type } & \\
\hline & \multicolumn{4}{|c|}{ Picture Recognition } & \multicolumn{4}{|c|}{ Face Recognition } \\
\hline & \multicolumn{2}{|c|}{ Young } & \multicolumn{2}{|c|}{ Elderly } & \multicolumn{2}{|c|}{ Young } & \multicolumn{2}{|c|}{ Elderly } \\
\hline & $\bar{M}$ & $S D$ & $M$ & $\overline{S D}$ & $\bar{M}$ & $\overline{S D}$ & $M$ & $S D$ \\
\hline Identical & .64 & .11 & .60 & 15 & .86 & .05 & .82 & .06 \\
\hline Changed & .36 & .10 & .38 & .11 & .78 & .08 & .77 & .07 \\
\hline New & .14 & .05 & .19 & .09 & .28 & .11 & .40 & .14 \\
\hline
\end{tabular}

Note-Picture-recognition judgments were hits with identical items and false alarms with changed and new items. Face-recognition judgments were hits with identical and changed items, and false alarms with new items.

between-subjects variable of age of subject and the withinsubjects variable of item type.

Both ANOVAs showed item-type main effects $\left[F(2,136)=392.5\right.$ and 678.5, $M S_{\mathrm{e}}=.009$ and .008 , for picture- and face-recognition judgments, respectively], as well as item-type $\times$ age-of-subject interactions $[F(2,136)$ $=3.53$ and $16.6, M S_{e}=.009$ and .008 , for picture- and face-recognition judgments, respectively]. ANOVAs of the picture-recognition proportions for each of the three item types showed a significant main effect of age only with new items $\left[F(1,68)=7.75, M S_{e}=.005\right.$ (other $p s>.10)$ ]. ANOVAs of the face-recognition proportions for each of the three item types showed significant main effects of age with new items $\left[F(1,68)=15.9, M S_{\mathrm{e}}=\right.$ $.016]$ as well as identical items $\left[F(1,68)=6.13, M S_{e}=\right.$ .013 ], though not with changed-expression items $(F<1)$. By the $\omega^{2}$ measure, subject age accounted for $18 \%$ of the variance in face-recognition judgments made in response to new items, $9 \%$ of the variance in picture-recognition judgments made in response to new items, $7 \%$ of the variance in face-recognition judgments made in response to identical items, and less than $1 \%$ of the variance elsewhere. Thus, the largest age difference was in facerecognition judgments made in response to new items (cf. Bartlett et al., 1989; Ferris et al., 1980; Smith \& Winograd, 1978).

Correlational analysis. For each of the 72 faces in the test, we computed the proportions of picture-recognition judgments and face-recognition judgments made by young and elderly subjects when the face served as an identical item, a changed item, and an entirely new item. We then computed correlations between each of these proportions and the proportions of familiar judgments from the corresponding age group in Experiment 1. Subjective familiarity was not significantly correlated with picturerecognition judgments, regardless of age group (Table 6 , two left columns). It was correlated with face-recognition judgments, regardless of item type, but only in the elderly group (Table 7, two left columns). Included in both tables are correlations for items that were consistently 
Table 6

Correlations between Picture-Recognition Judgments in Experiment 2 and Subjective-Familiarity Judgments in Experiment 1 for Young and Elderly Subjects Based on All Faces and on Faces Judged Consistently in Subjective Familiarity

\begin{tabular}{lccccc}
\hline & \multicolumn{2}{c}{ All Faces } & & \multicolumn{2}{c}{$\begin{array}{c}\text { Consistently } \\
\text { Judged Faces }\end{array}$} \\
\cline { 2 - 3 } \cline { 5 - 6 } Item Type & $\begin{array}{c}\text { Young } \\
(n=72)\end{array}$ & $\begin{array}{c}\text { Elderly } \\
(n=72)\end{array}$ & & $\begin{array}{c}\text { Young } \\
(n=34)\end{array}$ & $\begin{array}{c}\text { Elderly } \\
(n=38)\end{array}$ \\
\hline Identical & .16 & -.02 & & .32 & .04 \\
Changed & .05 & .15 & & .04 & .29 \\
New & -.12 & .16 & & -.08 & $.41^{*}$ \\
\hline
\end{tabular}

${ }^{*} p<.05$.

Table 7

Correlations between Face-Recognition Judgments in Experiment 2 and Subjective-Familiarity Judgments in Experiment 1 for Young and Elderly Subjects Based on All Faces and on Faces Judged Consistently in Subjective Familiarity

\begin{tabular}{lccccc}
\hline & \multicolumn{2}{c}{ All Faces } & & \multicolumn{2}{c}{$\begin{array}{c}\text { Consistently } \\
\text { Judged Faces }\end{array}$} \\
\cline { 2 - 3 } \cline { 6 - 7 } Item Type & $\begin{array}{c}\text { Young } \\
(n=72)\end{array}$ & $\begin{array}{c}\text { Elderly } \\
(n=72)\end{array}$ & & $\begin{array}{c}\text { Young } \\
(n=34)\end{array}$ & $\begin{array}{c}\text { Elderly } \\
(n=38)\end{array}$ \\
\hline Identical & .09 & $.33 \dagger$ & & .20 & $.46 \dagger$ \\
Changed & .06 & $.23^{*}$ & & .19 & $.46 \dagger$ \\
New & -.02 & $.37 \dagger$ & & -.01 & $.47 \dagger$ \\
\hline
\end{tabular}

${ }^{*} p<.05 . \quad \dagger p<.01$.

judged for subjective familiarity in Experiment 1 (as in Table 4). The pattern of correlations is substantially unchanged, though some of the $r$ are stronger.

\section{GENERAL DISCUSSION}

We conducted these studies to test an explanation for a puzzling phenomenon of aging and memory-an agerelated increase in false alarms in response to new faces. The explanation was couched in terms of a model (Bruce \& Young, 1986) in which it is claimed that face recognition is based on (at least) two processes-that of perceiving global resemblance, and that of recollecting contextual and semantic information. The hypothesis that we developed (Bartlett et al., 1991) holds that the elderly are deficient in recollecting contextual/semantic information, and/or in using such information to make recognition judgments, and so they rely heavily on resemblance information in recognizing faces. Since some new faces signal strong resemblance, a heavy reliance on resemblance information leads to many false alarms.

The context-recollection hypothesis makes an interesting prediction: elderly but not younger adults should show a positive correlation between judgments of subjective familiarity of faces and judgments that these faces have been previously seen in an experiment. This prediction was supported in Experiment 1, in which elderly but not young adults showed a positive correlation between subjective-familiarity judgments and false-recognition judgments across a set of 72 faces. Experiment 2 showed that the critical correlation generalized from false recognitions of new faces to correct recognitions of old faces. However, it did not generalize in a consistent way to correct recognitions of pictures of faces. The latter outcome is sensible, at least after the fact, since recognition memory for pictures of faces might be based largely on pictorial information, which is sharply distinguished from resemblance information in some face-processing models (Bruce \& Young, 1986).

Just as the findings supported a context-recollection hypothesis, they ruled against an alternative hypothesis, according to which age-related increases in false-alarm rates reflect age-related deficits in distinctiveness of encoding. This encoding-distinctiveness hypothesis did not predict the main result of the present study (that elderly but not young adults would show a correlation between familiarity judgments and false recognitions). Instead, it predicted a result that was not found (that elderly adults would exceed young adults in the strength of the correlation between face typicality and false recognitions).

Another alternative view of our findings posits age differences in contingent responding. The procedure that we used in Experiment 1 forced subjects to make two different judgments in response to each test item. Perhaps the elderly subjects had trouble with this dual-response task, and they adopted the strategy of making just a single judgment, and then generating two responses (subjective familiarity and recognition) on the basis of this single judgment. Hence, they tended to judge faces as: (1) both previously presented and subjectively familiar, or (2) neither previously presented nor subjectively familiar. The use of this strategy might produce a correlation between subjective familiarity and false recognition across the 72 faces, which is what we observed in the elderly group. However, contrary to the contingentresponse hypothesis, only 2 out of 17 young subjects and 3 out of 17 elderly subjects showed a statistically significant phi correlation between false-recognition and familiar judgments ( 2 young and 2 elderly subjects made no false recognitions or no familiar judgments, precluding the computation). Collapsing over subjects, the omnibus phi correlation between false-recognition and familiar judgments was -.013 for the young adult subjects versus .114 for the elderly subjects (total $n=1,368$ in each age group). The latter correlation was statistically significant $\left[\chi^{2}(1)=17.7, p<.01\right]$, as was the corresponding correlation computed across items (Table 4). However, it appears much too weak to support the hypothesis of contingent responding by elderly subjects.

Colleagues have argued that the low phi correlation in the elderly group might contradict our context-recollection hypothesis along with the contingent-response hypothesis. We beg to differ. The context-recollection hypothesis holds that older individuals tend to make relatively little use of contextual information, relying more heavily on resemblance information, in making recognition and familiar judgments. However, a subject who relies on 
resemblance information is in no way constrained to make recognition judgments in accordance with subjectivefamiliarity judgments or vice versa. When such a subject experiences resemblance in a face, he or she may simply guess among the three alternatives of seen before and familiar, seen before but unfamiliar, and not seen before but familiar. Alternatively, the subject may consider the strength of the resemblance in making a judgment (e.g. strong resemblance might lead to a judgment of seen before and familiar, whereas weaker resemblance might lead to a judgment of seen before but unfamiliar or not seen before but familiar). The point for present purposes is that a heavy reliance on resemblance information need not lead to a contingent relation between subjective-familiarity and recognition judgments. All we can predict is that the faces that evoke many recognition judgments should also evoke many familiar judgments. And this is what our findings revealed.

An issue calling for further discussion involves the troublesome task of inferring causality from simple correlations. It is an elementary fact that a correlation between subjective-familiarity and recognition judgments admits three interpretations: First, some factor closely linked to subjective familiarity might have an effect on recognition judgments. Second, some factor closely linked to recognition judgments might have an effect on subjective familiarity. Third, some third factor might affect both types of judgment.

Although we cannot yet decide among these three interpretations, we make the following two points: First, all three causal chains that are possible in principle are compatible with the context-recollection hypothesis. If the elderly are deficient in memory for context, or if they simply fail to use such memory in recognizing faces, one would expect not only that detection of resemblance with faces seen in life would affect recognition judgments, but also that detection of resemblance with prior test items would affect subjective-familiarity judgments. Moreover, other types of resemblance detection, such as matching with some type of face prototype (Bruce, 1988), might influence both judgments.

Second, the effects of test position on subjects' familiar judgments (Figure 2) add some support for one causal path, that of detection of resemblance with prior test items on familiar judgments in the elderly group. A plausible account for the test-position data is that the later a face occurred in the sequence, the greater the chances that it activated a trace of a face shown earlier in the sequence, and the less the chances that it activated a trace of a face encountered in everyday life. If so, and if subjects have good memory for context, faces occurring in later positions should get more false recognition judgments, but fewer familiar judgments. This is the pattern shown by young subjects (compare the filled triangles in Figures 1 and 2). However, if subjects have poor memory for context, faces that resemble prior faces in the test should be difficult to distinguish from faces that resemble other faces from "real life." Hence, faces that occur in later test po- sitions, and that frequently resemble prior faces in the test, might get more false-recognition judgments as well as more familiar judgments. This is the pattern shown by elderly subjects (compare the unfilled circles in Figures 1 and 2). The preceding argument assumes a causal effect of a factor related to false recognition on older subjects' familiar judgments.

A strictly empirical issue requires a brief comment. Table 3 shows that we found correlations between facetypicality and false-recognition judgments, and between false-recognition and subjective-familiarity judgments (in the elderly group), but that we did not replicate the previous finding of significant correlations between subjectivefamiliarity and typicality judgments (Bartlett et al., 1984; Vokey \& Read, 1988). Although future research must resolve this discrepancy, the face-age factor is plausibly involved. In the previous studies by Vokey and Read and Bartlett et al., only young faces were used, whereas here we used faces of all ages. Since the face-age variable had differing effects on familiar judgments and typicality ratings (Table 1), it becomes understandable that familiar judgments and typicality ratings appeared independent here. We note in addition that face familiarity and face typicality have been found to be uncorrelated in other investigations (Valentine \& Bruce, 1986).

In conclusion, in two experiments, we replicated the finding of an age-related increase in falsely recognizing faces. Moreover, in both studies, we found that elderly persons but not young adults showed a reliable correlation between: (1) false recognitions of the faces on a test and (2) judgments that these faces resemble faces known from life. This pattern was predicted on the basis of the hypothesis that the elderly suffer deficits in recollecting or using context, and that they place more reliance on resemblance information in making recognition judgments. From a broader perspective, our findings support models of face recognition (e.g., Bruce \& Young, 1986) that distinguish the processes of detecting resemblance (familiarity) from those of recollecting context. Indeed, in providing evidence that recollection of context is a factor in determining false-alarm rates, our findings serve to extend such models in a promising new way.

\section{REFERENCES}

Bartlett, J. C., Hurry, S., Thorley, W. (1984). Typicality and familiarity of faces. Memory \& Cognition, 12, 219-228.

Bartlett, J. C., Leslie, J. E. (1986). Aging and memory for faces versus single views of faces. Memory \& Cognition, 14, 371-381.

Bartlett, J. C., Leslie, J. E., Tubbs, A., \& Fulton, A. (1989). Aging and memory for pictures of faces. Psychology \& Aging, 4, 276-283.

Bartlett, J. C., Strater, L., \& Fulton, A. (1991). False recency and false fame of faces in young adulthood and old age. Memory \& Cognition, 19, 177-188.

Brown, E. L., Deffenbacher, K. A., \& Sturgill, W. (1977). Memory for faces and the circumstances of an encounter. Journal of Applied Psychology, 62, 311-318.

BRUCE, V. (1988). Recognizing faces. Hillsdale, NJ: Erlbaum.

Bruce, V., Young, A. W. (1986). Understanding face recognition. British Joumal of Psychology, 77, 305-327. 
Craik, F. I. M. (1977). Age differences in human memory. In J. E. Birren \& K. W. Schaie (Eds.), Handbook of the psychology of aging (pp. 384-420). New York: Van Nostrand Reinhold.

CraIK, F. I. M., \& McDowd, J. M. (1987). Age differences in recall and recognition. Journal of Experimental Psychology: Learning, Memory, \& Cognition, 13, 474-479.

Craik, F. I. M., \&imon, E. (1980). Age differences in memory: The roles of attention and depth of processing. In L. W. Poon, J. L. Fozard, L. S. Cermak, D. Arenberg, \& L. W. Thompson (Eds.), New directions in memory and aging: Proceedings of the George Talland Memorial Conference (pp. 95-112). Hillsdale, NJ: Erlbaum.

DAvies, G. M., SHePHERD, J. W., \& Elus, H. D. (1979). Similarity effects in face recognition. American Journal of Psychology, 92, 507-523.

Ferris, S. H., Crook, T., Clark, E., McCarthy, M., \& Rae, D. (1980). Facial recognition memory deficits in normal aging and dementia. Journal of Gerontology, 35, 707-714.

GoING, M., \& READ, J. D. (1974). The effect of uniqueness, sex of subject and sex of photograph on facial recognition. Perceptual Motor Skills, 39, 109-110.

Goldstein, A. G., StePhenson, B., \& Chance, J. (1977). Face recognition memory: Distribution of false alarms. Bulletin of the Psychonomic Society, 9, 416-418.

HANLEY, J. R., \& Cowell, E. S. (1988). The effects of different types of retrieval cues on the recall of names of famous faces. Memory \& Cognition, 16, 545-555.

HAY, D. C., Young, A. W. (1982). The human face. In A. W. Ellis (Ed.), Normality and pathology in cognitive functions (pp. 173-202). New York: Academic Press.

Light, L. L., Kayra-Stuart, F., \& Hollander, S. (1979). Recognition memory for typical and unusual faces. Journal of Experimental Psychology: Human Leaming \& Memory, 5, 212-228.

RANKIN, J. L., \& KAUSLER, D. H. (1979). Adult age differences in false recognitions. Journal of Gerontology, 34, 58-65.

SMITH, A. D. (1975). Partial learning and recognition memory in the aged. International Joumal of Aging \& Human Development, 6, 359-365.

SmITH, A. D., \& Winograd, E. (1978). Adult age differences in remembering faces. Developmental Psychology, 14, 443-444.
Valentine, T., \& Bruce, V. (1986). Recognizing familiar faces: The role of distinctiveness and familiarity. Canadian Joumal of Psychology, 40, 300-305.

VOKEY, J. R., \& READ, J. D. (1988). Typicality, familiarity, and recognition of male and female faces. Canadian Journal of Psychology, 42, 489-495.

Winograd, E. (1981). Elaboration and distinctiveness in memory for faces. Joumal of Experimental Psychology: Leaming, Memory, \& Cognition, 7, 181-190.

YounG, A. W., HAY, D. C., ELLIS, A. W. (1985). The faces that launched a thousand slips: Everyday difficulties and errors in recognizing people. British Journal of Psychology, 76, 495-523.

\section{NOTES}

1. In most prior work on familiarity judgments, the subjects' task has been to distinguish faces of persons they know from faces of persons they have never seen before. In contrast, all of the faces in the Vokey and Read (1988) study, and all of the faces in Experiment 1, were unknown to the subjects. To prevent confusion, we will refer to the judgments subjects made in these tasks as subjective-familiarity judgments.

2. Whereas Vokey and Read (1988) had subjects make typicality, familiarity, and recognition judgments simultaneously, with only one pass through the list, we were concerned that this procedure would be too taxing for the elderly.

3. This result suggests either a bias to judge young female and old male faces as familiar, or a greater prevalence of such faces in the memories of our subjects (possibly due to cultural/ecological factors affecting viewing or encoding of faces in life).

4. We have inverted the 7-point typicality scale so that higher numbers mean greater typicality. We note that the typicality ratings by young versus elderly subjects were correlated at $r=.70$.

5 . With $70 \mathrm{df}$, an $r$ of .23 gives $p<.05$, and an $r$ of .30 gives $p<.01$.

(Manuscript received June 4, 1990; revision accepted for publication October 24, 1990.) 